\title{
POWER DISTRIBUTION FOR THE ATLAS LAR TRIGGER DIGITIZER BOARD
}

MASSIMO LAZZARONI (UNIVERSITÀ DEGLI STUDI DI MILANO/INFN-MI)
ON BEHALF OF THE ATLAS LIQUID ARGON CALORIMETER GROUP

The research activity for the design of the power distribution
section of the ATLAS LAr Trigger Digitizer Board board (LTDB) will be presented. Many aspects concerning the radiation hardness and the ability to operate Point-of-load converters even in presence of high magnetic fields will be covered.
Devices designed by CERN have been used and their Devices designed by CERN have been used and their
capability for implementation on the ATLAS LTDB has been exploited with the aim to have a power distribution section with the required performances.

\section{Introduction}

A Liquid-argon Trigger Digitizer Board (LTDB) is being Argon Calorimeters.

Several ASICs such as GBTX, optical transmitter/receivers, level translators and custom designed ADCs, as well as multiple ARTIC FPGAs from Xilinx are mounted on the board to several different supply voltages and a well-specified power-up sequence. Furthermore, dfe

The full functionality of the board requires considerable LTDB has to operate in a hostile environment. Each component has to be tested for radiation and magnetic field tolerance, an special design techniques have to be applied. The most
important aspects are basically those related to the reliability of the devices over time.

Supply circuits are particularly affected by hostile environment. For example, high magnetic fields can lead to malfunctions, failures and degradation of the performances of some switching power supplies. It was therefore considered useful to study these aspects carefully with the aim of being able to desig power systems that can work reliably on the ATLAS detector.
We propose the use of Point of Load (PoL) developed by This device is able to assure optimal performance in the presence of both high magnetic fields and high level of voltages between $1 \mathrm{~V}$ and $4 \mathrm{~V}$ and up to $10 \mathrm{~W}$ of power each
The considered PoL provides a maximum output current $(4 \mathrm{~A})$ and maximum deliverable power of $10 \mathrm{~W}$. Only for the digital part of the LTDB board 23 FEASTMPs are therefore needed part of the LTDB board 23 pifte this large number of devices.

In what concern the analog part of the LTDB, LDOs have been proposed but this aspect will be not covered here. ard devices, a supply voltage with a low level of residual ripple (less than 10 considered that the ripple may be affected by the value of the input voltage, by the value of the output voltage and, finally, by portant requirements.

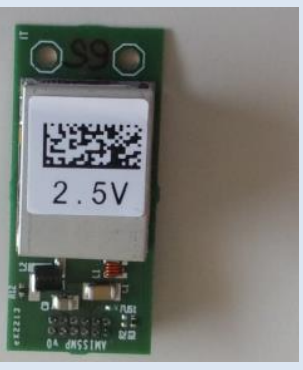

\section{FEASTMP PoL Features}

2.1 Ripple: Output behavior in terms of noise is an by means of the ripple in the outpul signal. lis important to variation of the output of a device which has been derived rom an alternating current source. The value of the output ipple is very important in many situations. In the LTDB a ipple less than $10 \mathrm{mVpp}$ is required. The ripple of the system depends also on the magnetic field strength. For device under conditions stronger than the standard. Som dex or different input voltage. Measurements have been manufacturer.

In the present case the PoL is a DC-DC converter and it application magnetic field but not in a significant way. The experimental results are perfectly in compliance with the values given by the manufacturer (see the following figures) assure the functionalities of the LTDB. These devices require relative large area of the boer distribution. For this reason a CERN and the use of Point of Load (POL) developed by radiations. The digital components on the LTDB require

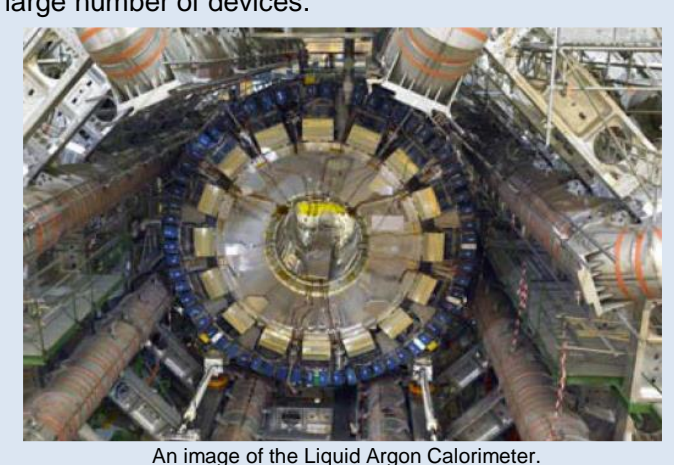

Some preliminary tests confirm that the FEASTM can comply theresting key point. Actually, the noise can be evaluated the LTDB board in its environment the value will be $\sim 0.6 T$ obtained with conditions equal to the ones indicated by the

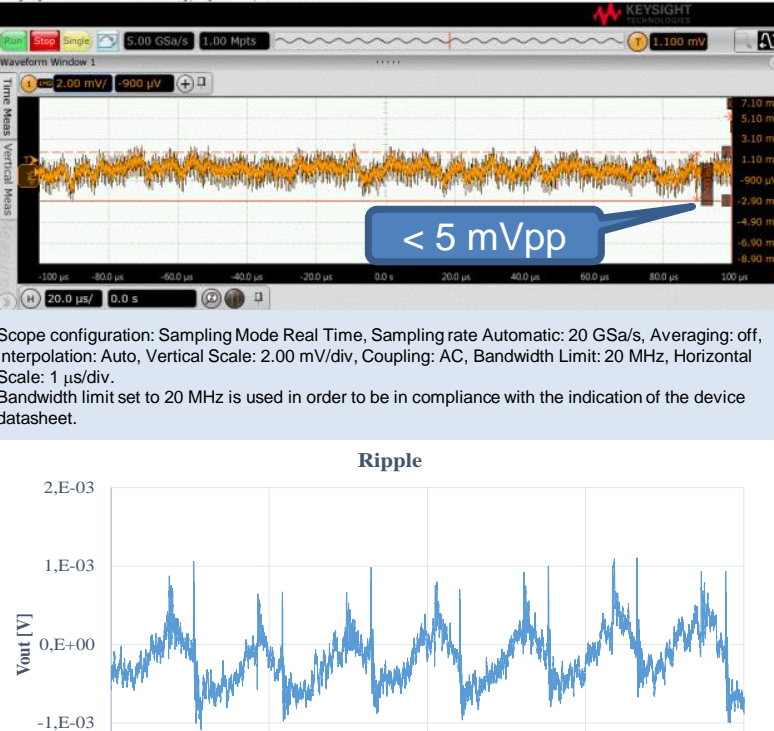

It is important to verify the performance of this device with different input voltage because the main converter used in ATLAS - LAr (LAr Calorimeter) is designed with many output voltage ripple is very important and shown in the above figure.

2.2 Noise: Noise is also important in the considered applicalions. The spectrum highlights the main frequency which is used by the converter (at

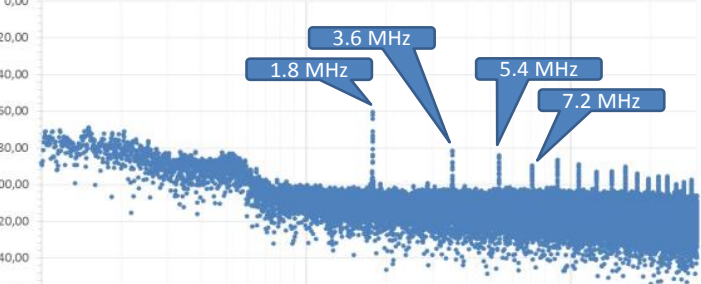

2.3 Voltage levels: The usableoutput voltages of the actua The FASTMP ) are: $11 \mathrm{~V}, 7 \mathrm{~V}, 6 \mathrm{~V}, 4 \mathrm{~V}$.

with positio in POL dove, up to now, operates only denoted SEASTMN will be available in the version, The input voltage of $4 \mathrm{~V}$ is not suitable to be used as input for FEASTMP supply. FEASTMP are, in fact, designed for an input range $5-12 \mathrm{~V}$.

2.4 Radiation Tolerance: TID up to $>200 \mathrm{Mrad}(\mathrm{Si})$ displacement damage up to $5 \times 10^{14} \mathrm{n} / \mathrm{cm}^{2}(\mathrm{TM}$.

$>65 \mathrm{MeV} \mathrm{cm}^{\wedge} 2 / \mathrm{mg}$ (only short SETs smaller than $20 \%$ of the nominal Vout are observed). See ref. [1 -3$]$ for details.

2.5 Magnetic field tolerance: in excess of 40,000 Gauss. Experimental tests were conducted with
values typical for installed LTDB board.

Here are reported, for example, the results obtained with Vin $=12 \mathrm{~V}$, Vout $=1.8 \mathrm{~V}$ and lout $=3.2 \mathrm{~A}$ and magnetic Field test conditions as in following Table, according to the rising and falling of the magnetic field as indicated by
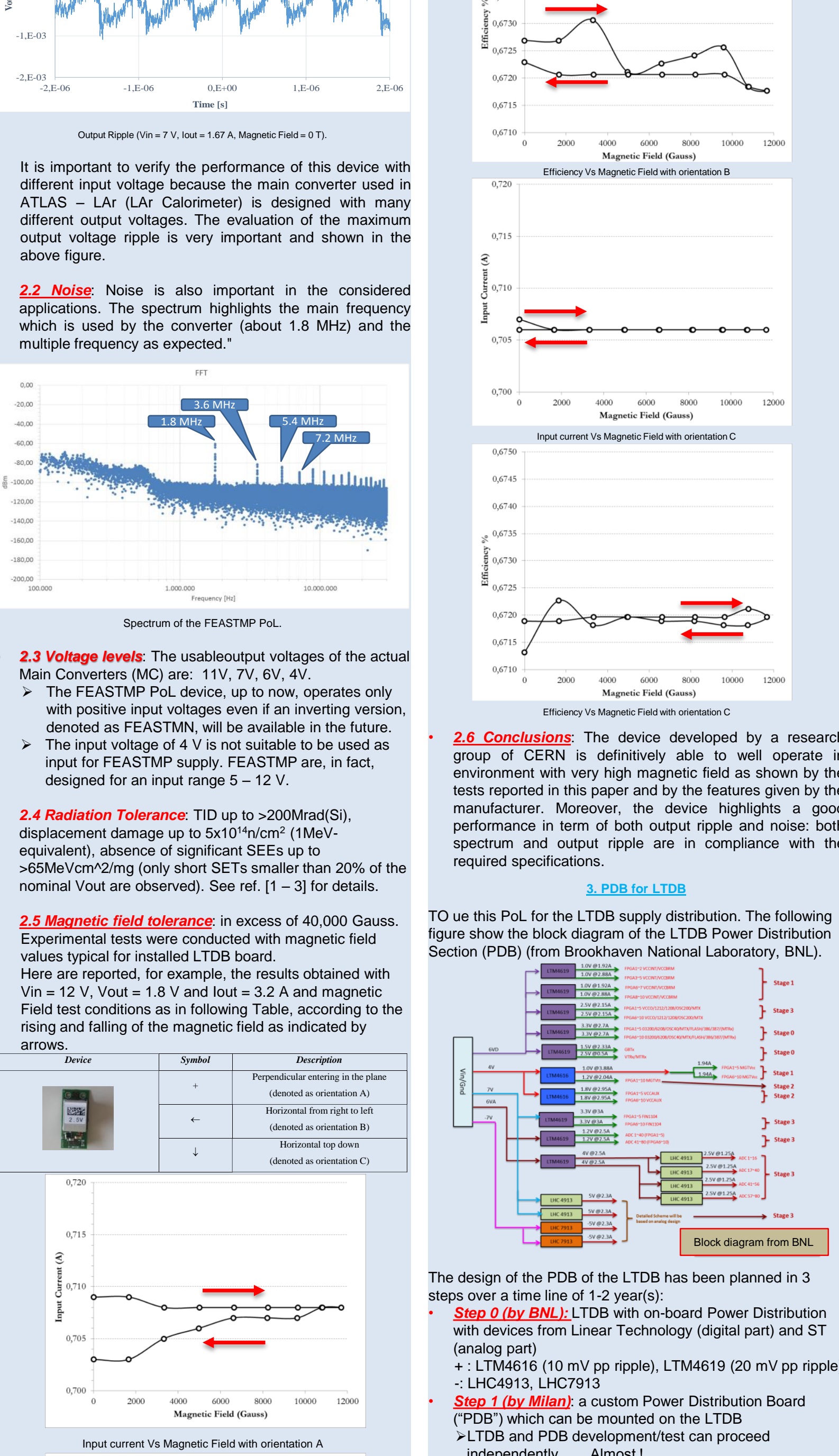

The design of the PDB of the LTDB has been planned in 3

Step 0 (by BNL).LTDB with on-board Power Distribution with devices from Linear Technology (digital part) and ST

(analog part)

Step 1 (by Milan): a custom Power Distribution Board TTDB and PDB development/test can proced independently ..... Almost!
$>$ Performance tests on the PDB as a system are possible Different PoL devices can be tested (if the interconnections between the two boards are interconnel
changed)
Step 2 (by Milan): Power Distribution Section as a part of

pDB layout "integrated" into the

Interesting for final LTDB area)

The design is based on FEAST2 ASICs and re-desig Untested ASICs (expected yield 95\%) already

purchased.

CERN is looking into the possibility of delivering full

4. The Step 1

The Step 1 consist in the design and manufacture of the PDB board. Many issues: mechanical, electrical etc.

Max current delivered by FEASTMP and its efficiency:
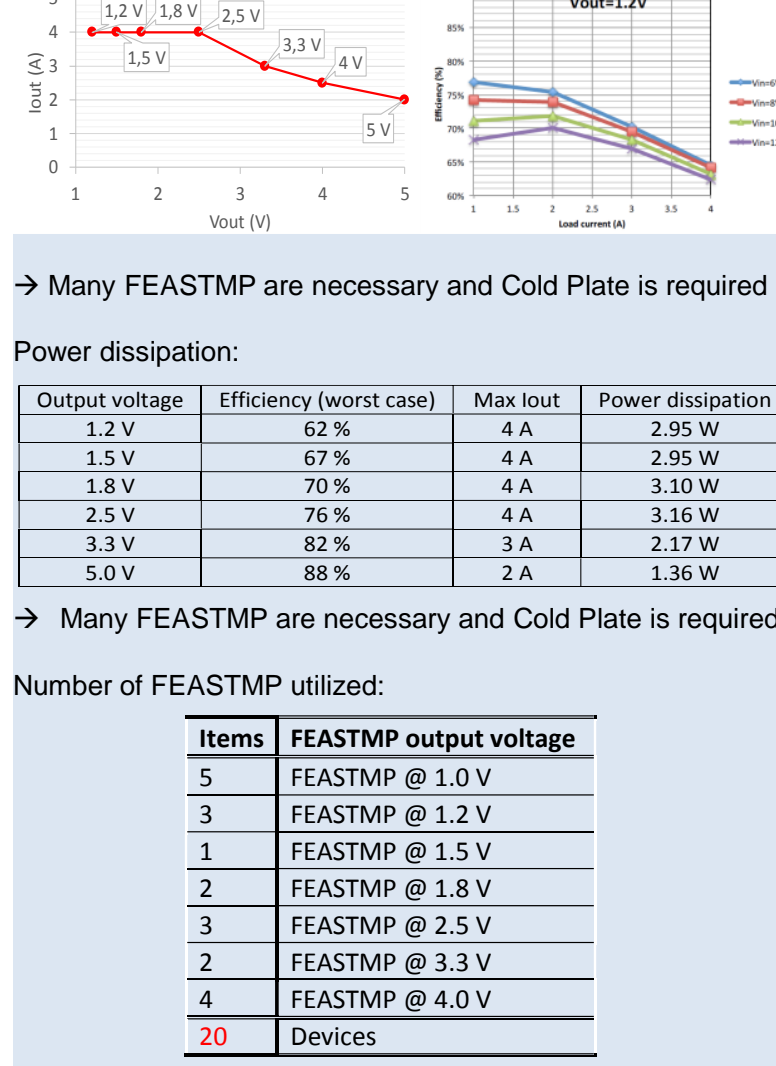

PCB Design difficulties

$\rightarrow$ many openings are necessary
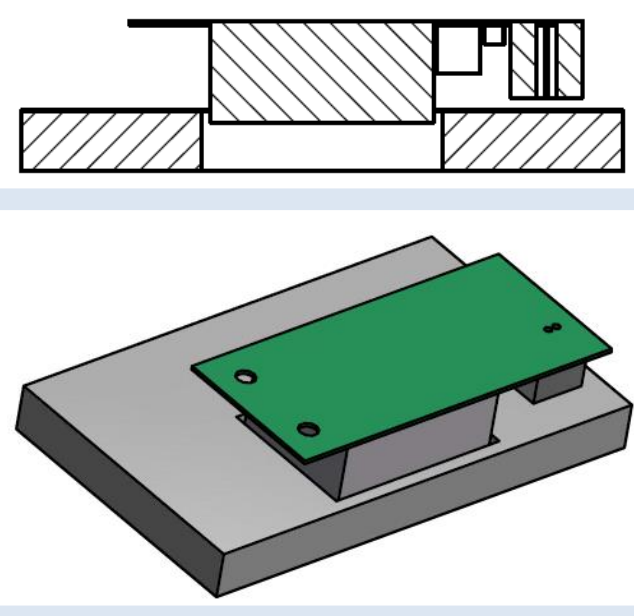

Reduced available area Max: $410.55 \times 53-74 \mathrm{~mm}$ (she linear devices (LDO) on the PDB.

Therefore the analog voltages will be obtained with the TTEP 1 is rocus moun od on the LTDB.

LDP 1 is foused on underslanding the FEASTMP 作

\section{Conclusions}

The design of the PDB has been addressed and the layout is now complete.

Table of connections between LTDB and PDB as been defined (with BNL)

Final PCB is reported in the following figure. PCB is in production:

Tests expected in Nov/Dec 2015;

If test successful the PDB will be inserted and tested as part of the LTDB by Brookhaven National Laboratory (BNL).

[1] Michelis, S.; Faccio, F.; Jarron, P.; Kayal, M., "Air core inductors study for DCIDC power supply in harsh radiation environment," in Circuits and Systems International IEEE Northeast Workshop on, pp. 105-108, 22-25 June 2008.
[2] Michelis, S.; Allongue, B.; Blanchot, G.; Buso, S.; Faccio, F.; Fuentes, C.F

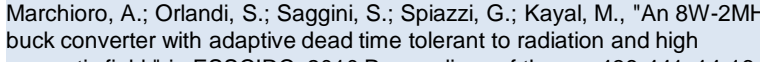

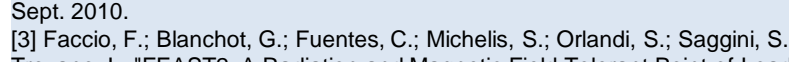

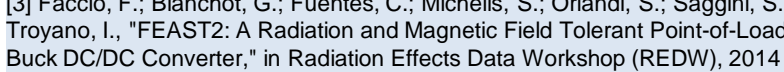

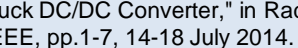

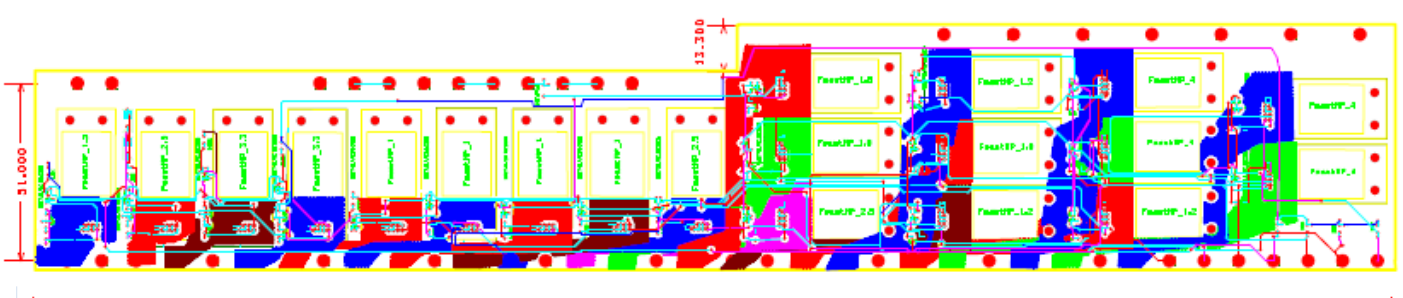

